\title{
Knockdown of Notch1 inhibits nasopharyngeal carcinoma cell growth and metastasis via downregulation of CCL2, CXCL16, and uPA
}

\author{
Huajiao Guo ${ }^{1}$ | Fuhao Wang ${ }^{2}$ | Yuwen Diao ${ }^{2}$ Zhe Zhang ${ }^{3}$ | Qiuyan Chen ${ }^{4}$ |

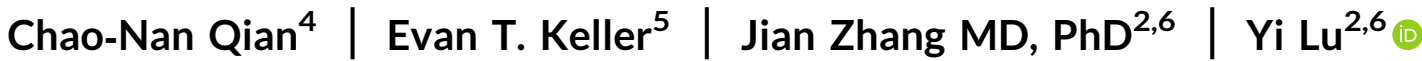

${ }^{1}$ Department of Oncology, Beihai People's Hospital, Beihai, Guangxi, China

${ }^{2}$ School of Medicine, Southern University of Science and Technology, Shenzhen,

Guangdong, China

${ }^{3}$ Department of Otolaryngology, First Affiliated Hospital of Guangxi Medical University, Nanning, Guangxi, China

${ }^{4}$ Department of Nasopharyngeal Carcinoma, Sun Yat-sen University Cancer Center,

Guangzhou, Guangdong, China

${ }^{5}$ Department of Urology and Pathology, School of Medicine, University of Michigan, Ann Arbor, Michigan

${ }^{6}$ Guangdong Provincial Key Laboratory of Cell Microenvironment and Disease Research, Shenzhen, Guangdong, China

\section{Correspondence}

Jian Zhang and Yi Lu, School of Medicine, Southern University of Science and Technology, No. 1088 Xue Yuan Blvd, Shenzhen, 518055 Guangdong, China. Email: zhangjian@sustc.edu.cn (JZ) and luy3@sustc.edu.cn (YL)

Funding information

Science and Technology Foundation of Shenzhen Grants, Grant/Award Numbers: JCYJ2017030711041760,

JCYJ20170307105128101; National Natural Science Foundation of China, Grant/Award Numbers: 81130046, 81773146; Guangdong Provincial Key Laboratory of Cell

Microenvironment and Disease Research, Grant/Award Number: 2017B030301018

\begin{abstract}
Notch pathway is a highly conserved cell signaling system that plays very important roles in controlling multiple cell differentiation processes during embryonic and adult life. Multiple lines of evidence support the oncogenic role of Notch signaling in several human solid cancers; however, the pleiotropic effects and molecular mechanisms of Notch signaling inhibition on nasopharyngeal carcinoma (NPC) remain unclear. In this study, we evaluated Notch1 expression in NPC cell lines (CNE1, CNE2, SUNE1, HONE1, and HK1) by real-time quantitative PCR and Western blot analysis, and we found that CNE1 and CNE2 cells expressed a higher level of Notch1 compared with HONE1, SUNE1, and HK1 cells. Then Notch1 expression was specifically knocked down in CNE1 and CNE2 cells by Notch1 short hairpin RNA (shRNA). In Notch1 knockdown cells, cell proliferation, migration, and invasion were significantly inhibited. The epithelial-mesenchymal transition of tumor cells was reversed in Notch1-shRNA-transfected cells, accompanied by epithelioid-like morphology changes, increased protein levels of E-cadherin, and decreased expression of vimentin. In addition, knockdown of Notch1 markedly inhibited the expression of urokinase plasminogen activator (UPA) and its receptor UPAR, and chemokines $\mathrm{C}-\mathrm{C}$ motif chemokine ligand 2 and $\mathrm{C}-\mathrm{X}-\mathrm{C}$ motif chemokine ligand 16 , indicating that these factors are downstream targets of Notch1. Furthermore, deleting uPA expression had similar effects as Notch1. Finally, knockdown of Notch1 significantly diminished CNE1 cell growth in a murine model concomitant with inhibition of cell proliferation and induction of apoptosis. These results suggest that Notch1 may become a novel therapeutic target for the clinical treatment of NPC.

KEYWORDS

CCL2, EMT, nasopharyngeal carcinoma, Notch1, uPA
\end{abstract}

Abbreviations: CCL2, C-C motif chemokine ligand2; CM, conditioned media; CXCL16, C-X-C motif chemokine ligand 16; ECL, enhanced chemiluminescence; ELISA, enzyme-linked immunosorbent assay; EMT, epithelial-mesenchymal transition; Hes-1, hairy and enhancer of split-1; NICD, notch intracellular domain; NICD-Ov, NICD overexpression; NPC, nasopharyngeal carcinoma; RT, room temperature; uPA, urokinase plasminogen activator; uPAR, UPA receptor. 


\section{1 | INTRODUCTION}

Nasopharyngeal carcinoma (NPC) is a disease that is rare in most parts of the world, but is very common in both southern China with an incidence of 10 to 20/100 000 population per year, as well as in other Southeast Asian nations. ${ }^{1,2}$ With properties of special anatomic location, and sensitivity to radiotherapy and chemotherapy, the first-line for NPC treatment in clinics is radiotherapy. ${ }^{3,4}$ However, radiotherapy is only suitable for patients with primary tumors and localized tumors with regional lymph node metastases. Relapse or formation of distant metastases often occur after radiotherapy, resulting in a 5-year survival rate of about 50 to $60 \%{ }^{5}$ In addition, any patients with NPC develop resistance to radiotherapy that correlates with poor prognoses. ${ }^{6}$ Therefore, the identification of novel targets and validation of potential therapeutic approaches are urgently needed.

The Notch signaling pathway is a highly evolutionarily conserved pathway that is involved in pleiotropic functions, such as regulating cell differentiation, proliferation, apoptosis, and ultimately cell fate. Notch signaling was first identified in a study of Drosophila with serration wings by Morgan's group in 1917. ' Four Notch receptors, namely, Notch1, Notch2, Notch3, and Notch4, and five ligands, namely, Jagged1, Jagged2, Delta-like ligand 1 (DII1), DII3, and DII4, have been described in mammals. ${ }^{8,9}$ In the last few decades, progress has been made in both basic and clinical studies of Notch signaling, not only in developmental biology but also in cancer stem cell biology. ${ }^{10,11}$ Aberrant expression of Notch signaling proteins has been reported in a wide variety of human cancers, including T-cell acute lymphoblastic leukemia, the first cancer type in which Notch 1 was described as a factor that promotes cancer development. ${ }^{12}$ The potential mechanism of Notch signaling has recently been explored in breast cancer, ${ }^{13-15}$ ovarian cancer, ${ }^{16,17}$ renal cell carcinoma, ${ }^{18}$ and melanoma. ${ }^{19}$ The overexpression of Notch receptors and ligands in cancer correlates with a poorer prognostic profile and lower survival rates. ${ }^{20}$ However, it has also been shown that Notch signaling has growthsuppressive functions, suggesting that this pathway is highly contextdependent. The most representative evidence for Notch as a tumor suppressor is in mice with skin cancer. ${ }^{21}$ In addition, it has been shown that Notch has a growth-promoting function in non-small cell lung cancer (NSCLC), ${ }^{22,23}$ whereas constitutively active Notch1 and Notch2 cause profound growth arrest in small cell lung cancer (SCLC). ${ }^{24}$ It was recently reported that microRNA $139-5 p$ reverses the Notch1-mediated epithelial-mesenchymal transition (EMT) in glioma. ${ }^{25}$ Despite these advances, little information regarding Notch signaling in NPC is available, and the molecular mechanism of the Notch pathway in this disease is poorly defined. Thus, in this study, we investigated the role of Notch1 in NPC onset and progression.

\section{2 | MATERIALS AND METHODS}

\section{1 | Reagents and antibodies}

Antibodies against Notch1 (\#3608), E-cadherin (\#3195), Vimentin (\#5741), $\beta$-catenin (\#8480), Slug (\#9585), claudin-1 (\#13255),
Caspase3 (\#9662), Caspase9 (\#9502), Bcl2 (\#4223), cyclin E (\#20808), and cyclin D (\#2978) were purchased from Cell Signaling Technology (Boston, MA). Anti-human monocyte chemoattractant protein 1 (MCP-1/CCL2, \#MAB279), C-X-C motif chemokine ligand 16 (CXCL16, \#MAB976), urokinase plasminogen activator (UPA; \#MAB1310), and UPA receptor (UPAR; \#MAB807) monoclonal antibodies were purchased from R\&D Systems (Minneapolis, MN). All the chemical reagents and $\beta$-actin monoclonal antibody were purchased from Sigma (St. Louis, MO).

\section{2 | Cell culture}

The human NPC cell lines, CNE1, CNE2, HK1, HONE1, and SUNE1, were kindly provided by Dr. Chao-Nan Qian (Sun Yat-sen University Cancer Center, Guangzhou, China) and cultured in Dulbecco's Modified Eagle's Medium (DMEM; Invitrogen, Carlsbad, CA) supplemented with $10 \%$ fetal bovine serum (FBS) and $1 \%$ penicillinstreptomycin solution (Invitrogen). CNE1 and HK1 cells are welldifferentiated tumor cells, whereas CNE2, HONE1, and SUNE1 cells are poorly differentiated tumor cells. ${ }^{26,27}$ Human nasal epithelial cells (HNEC) were purchased from Promocell GmbH (Heidelberg, Germany) and cultured in Airway Epithelial Cell growth medium (Promocell $\mathrm{GmbH}$ ). All the cell lines were grown in a humidified incubator containing $5 \% \mathrm{CO}_{2}$ at $37^{\circ} \mathrm{C}$ and tested to rule out mycoplasma contamination.

\section{3 | Conditioned medium}

Conditioned medium (CM) was harvested from CNE1 and CNE2 cell lines. Briefly, $2 \times 10^{6}$ cells were plated overnight in a $10-\mathrm{cm}$ dish in DMEM supplemented with $10 \%$ FBS. The next day, cells were washed twice with phosphate-buffered saline (PBS) and the medium was changed to DMEM with $1 \%$ FBS. The CM was collected after 48 hours. To normalize for differences in cell density because of proliferation during the cell culture period, cells from each well were collected and the total DNA content/well was determined. Then, CM was normalized for DNA content between samples by adding DMEM.

\section{4 | Notch1-shRNA transfection}

shRNA molecules specific to Notch1 (sense 5'-CCGGGCATGGTGCC GAACCAATACACTCGAGTGTATTGGTTCGGCACCATGCTTTTTTG-3'; antisense 5'-AATTCAAAAAGCATGGTGCCGAACCAATACACTCGAG TGTATTGGTTCGGCACCATGC-3') were designed from homo sapiens Notch1 complete mRNA (Accession No: NM_017617.3, position 6950-6970) using Block-iT RNAi Designer (Invitrogen). The doublestranded shRNA oligo was annealed and inserted into pLKO.1 lentiviral vector (Addgene, Cambridge, MA) containing the puromycin resistance gene. Then, the recombinant construct was verified by sequencing (Invitrogen). Lentiviral particles were assembled by transiently co-transfecting $293 \mathrm{~T}$ cells with the shRNA-expressing lentiviral vector, and packaging the plasmid $p R R E$, vesicular stomatitis 
virus envelope plasmid pMD2G, and gene transfer plasmid RSV-REV. CNE1 and CNE2 cells were infected with lentiviral particles (Notch1shRNA and empty vector [EV], respectively) in the presence of $8 \mu \mathrm{g} / \mathrm{mL}$ polybrene (Sigma). Two days after infection, cells were selected by adding puromycin (Sigma) in culture medium and maintained for at least 2 weeks.

\section{5 | uPA-shRNA transfection}

The shRNA molecules specific to uPA (sense 5'-CCGGGCTGA GTTTCCTGGACTTAGTCTCGAGACTAAGTCCAGGAAACTCAGCTT TTTG-3'; antisense 5'-AATTCAAAAAGCTGAGTTTCCTGGACTTAGT CTCGAG ACTAAGT CCAGGAAACTCAGC-3') were designed from homo sapiens uPA complete mRNA (Accession No: NG_011904.1, position 1784-1804) using Block-It RNAi Designer. The procedure of UPA-shRNA construct and transfection please see the above (Notch1-shRNA transfection).

\section{6 | Real-time quantitative PCR}

Total RNA was extracted with TRIzol reagent (Invitrogen), and cDNA was synthesized from $2 \mu \mathrm{g}$ total RNA using the SuperScript III FirstStrand Kit (Invitrogen). The reverse transcription reaction was conducted at $65^{\circ} \mathrm{C}$ for 5 minutes followed by $50^{\circ} \mathrm{C}$ for 50 minutes and $70^{\circ} \mathrm{C}$ for 15 minutes. PCR primers for Notch1 consisted of forward 5'-GGCACTTTCTGTGAGGAGGA-3' and reverse 5'-GCAGTCAGGCGT GTTGTTCT-3'. PCR primers for uPA consisted of forward 5'-AAC TCTGCCACTGTCCTTC-3' and reverse 5'-CGGTTGTCTGGGTTCC TG-3'. PCR primers for hairy and enhancer of split-1 (Hes1) consisted of forward 5'-AGCTCGCGGCATTCCAAG-3' and reverse 5'-AGCG GGTCACCTCGTTCA-3'. PCR Primers for glyceral-dehyde3-phosphate dehydrogenase (GAPDH) consisted of forward 5'-AGCCACATCGCT CAGACA-3' and reverse 5'-GCCCAATACGACCAAATCC-3'. GAPDH was used as an internal control. Real-time quantitative PCR (qPCR) was performed as previously described. ${ }^{28,29}$ The relative expression of Notch1 or uPA to GAPDH was calculated using the $\triangle \mathrm{CT}$ method. ${ }^{30}$

\subsection{Western blot analysis}

Cell lysates were prepared using standard procedures. ${ }^{31}$ The membranes were incubated at $4^{\circ} \mathrm{C}$ overnight with Notch1, E-cadherin, vimentin, $\beta$-catenin, Slug, claudin-1, cyclin E, and cyclin D primary antibodies (Cell Signaling Technology). After being washed in TBST, the membranes were incubated with the horseradish peroxidase-conjugated secondary antibody, and the proteins were detected using the enhanced chemiluminescence detection system (Thermo Fisher Scientific, Waltham, MA). As a control for equal protein loading, $\beta$-actin (Sigma) was visualized.

\section{8 | Enzyme-linked immunosorbent assay}

To measure the protein levels of $\mathrm{C}-\mathrm{C}$ motif chemokine ligand 2 (CCL2), CXCL16, UPA, and UPAR in CM collected from CNE1 and CNE2 wild-type cells, the cells transfected with EV or Notch1-shRNA was subjected to enzyme-linked immunosorbent assay (ELISA; R\&D Systems). ELISAs were performed according to the manufacturer's instructions.

\subsection{Cell migration and invasion assay}

The cells were seeded in a 24-well matrigel invasion chamber (BD Biosciences, Bedford, MA), and cell migration and invasion assays were conducted as previously described. ${ }^{28}$ After 24 hours, the cells in the upper chamber were removed, and cells that invaded through the matrigel matrix membrane were stained with crystal violet after being fixed in paraformaldehyde. Then, the numbers of cells that penetrated the membrane in 10 microscopic fields were quantified by counting at a 200x magnification per filter. The invasive ability was defined as the proportion of cells that penetrated the Matrigelcoated membrane to the number of cells that migrated through the uncoated membrane (baseline migration).

\subsection{0 | Cell proliferation assay}

Cell proliferation was measured using the CellTiter 96 AQeous Nonradioactive Cell Proliferation Assay (Promega, Madison, WI) according to the manufacturer's instructions. Briefly, the cells were plated in 96-well plates at a density of 2000 cells/well, in $100 \mu \mathrm{L}$ DMEM containing $10 \% \mathrm{FBS}$. The cells were incubated at $37^{\circ} \mathrm{C}$ in a humidified $5 \% \mathrm{CO}_{2}$ atmosphere for 24,48 , and 72 hours and then, $20 \mu \mathrm{L}$ MTS solution was added. After incubation for 2 hours at $37^{\circ} \mathrm{C}$, the absorbance of each well at $490 \mathrm{~nm}$ was recorded by an ELISA plate reader.

\subsection{1 | Terminal deoxynucleotidyl transferase dUTP nick-end labeling assay}

The terminal deoxynucleotidyl transferase dUTP nick-end labeling (TUNEL) assay was performed with the In Situ Cell Death Detection kit (Roche, Indianapolis, IN) according to the manufacturer's instructions. Briefly, the cells were plated in an 8-chamber Poly-L-Lysine vessel tissue culture-treated glass slide (BD Biosciences), cultured for 48 hours, and fixed in 4\% paraformaldehyde for 1 hour at room temperature (RT). After washing with PBS, the cells were incubated in blocking buffer $\left(3 \% \mathrm{H}_{2} \mathrm{O}_{2}\right.$ in methanol) for 15 minutes at RT. Then, the cells were rinsed with PBS and incubated in a permeable solution ( $0.1 \%$ Triton $\mathrm{X}-100$ in $0.1 \%$ sodium citrate, freshly prepared) for 2 minutes on ice. Next, the cells were incubated with TUNEL reaction mixture for 1 hour at $37^{\circ} \mathrm{C}$ in a humidified chamber, followed by restaining with Hoechst 33342 (Sigma). As a positive control, fixed and permeabilized cells were treated with DNase I (Sigma) for 10 minutes; as a negative control, fixed and permeabilized cells were incubated with label solution only. The coverslips were mounted with PBS containing Antifade Mounting Medium (Vector Lab, Burlingame, CA) and pictures were taken under a fluorescence microscope. The percentage of apoptotic cells with DNA nick end-labeling was measured by counting cells exhibiting green fluorescent nuclei at $200 \times$ in 10 randomly chosen fields in triplicate plates. 


\subsection{2 | In vivo tumor models}

The animal protocol was approved by the Institutional Animal Care and Use Committee (Guangxi Medical University, Guangxi, China). Nude mice were from Animal Central of Guangxi Medical University and housed under specific pathogen-free conditions in accordance with the National Institute of Health guidelines. Thirty nude mice were used in these experiments (age: 5-6 weeks). CNE1 wild-type cells, and cells transfected with Notch1-shRNA or EV were subcutaneously injected into the right flank of the mice (n $=10 \mathrm{mice} /$ group). Tumor growth was monitored when it was palpable, and two perpendicular axes were measured using a caliper twice a week. Tumor volume was calculated using the formula: volume $=$ length $\times$ width $^{2} / 2 .^{32,33}$ The tumor cells were allowed to grow for 4 weeks, at which time the mice were killed, and the tumor tissue was collected.

\subsection{3 | Statistical analysis}

Numerical data are expressed as mean \pm standard deviation (SD) or mean \pm standard error of the mean (SEM), as indicated in the figure legends. The calculations were done using the Stat View system (Abacus Concepts, Berkeley, CA). One-way analysis of variance was used for all the studies. Fisher's least-significant difference was used for the post-hoc analysis. $P<.05$ was statistically significant.

\section{3 | RESULTS}

\section{1 | Notch1 expresses significantly higher levels in NPC tissues and cell lines}

The analysis of the gene expression data sets from Gene Expression Omnibus (GEO) database ${ }^{34}$ revealed that Notch1 expression levels were significantly higher in NPC tissues than in normal nasopharyngeal tissues (Figure 1A). To confirm that the Notch1 pathway plays key roles in NPC development, we measured Notch1 expression levels in normal nasal epithelial cells (HNEC) and the NPC cell lines, CNE1, HK1, CNE2, HONE1, and SUNE1, by qPCR and Western blot analysis. We found that all NPC cell lines (CNE1, HK1, CNE2, HONE1, and SUNE1) expressed Notch1 gene and protein (Figure 1B,C) at higher levels compared with the HNECs. Notch1 protein levels in CNE1 and CNE2 cells were much higher than in other NPC cell lines, prompting us to select these two cell lines for subsequent studies.

\subsection{Knockdown of Notch1 expression inhibits NPC cell growth and induces cell apoptosis}

To investigate whether Notch1 might be an effective therapeutic target for NPC, we used shRNA to knockdown Notch1 gene expression in CNE1 and CNE2 cells. qPCR and Western blot analysis were conducted to confirm the knockdown efficiency. Notch1 expression was reduced by over $80 \%$ in cells transfected with Notch1-shRNA
(A)

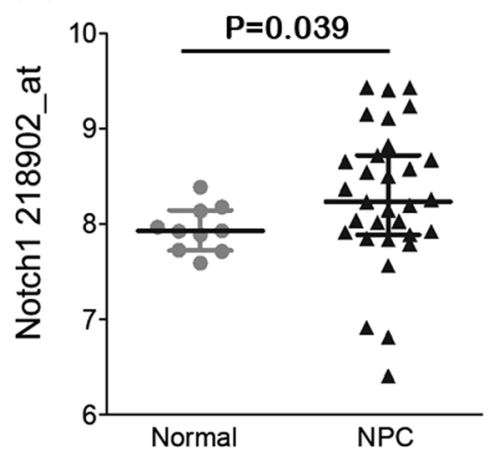

(B)

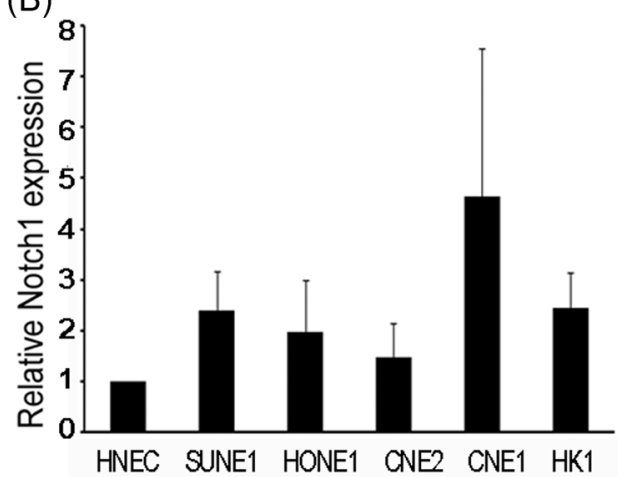

(C)
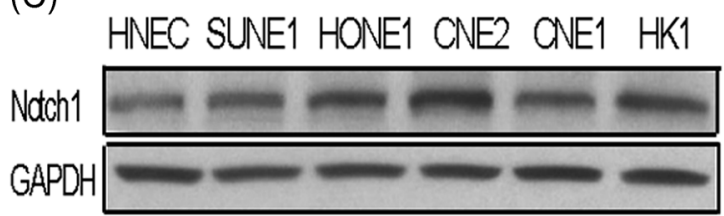

FIGURE 1 Constitutive expression of Notch1 in NPC tissues and cell lines. A, The scatterplot shows that the Notch1 gene was highly expressed in NPC tissues by Human Genome U133 Plus 2.0 Array. ${ }^{34}$ Total RNA extracted from laser-captured epithelium from 31 NPCs and 10 normal healthy nasopharyngeal tissue specimens. Sample data includes T stage, N stage, and race/ethnicity. B, Notch1 expression in HNEC, SUNE1, HONE1, CNE2, CNE1, and HK1 cells was measured. Total RNA was extracted from the indicated cells and Notch1 mRNA expression was detected by qPCR. C, Cell lysates collected from HNEC, SUNE1, HONE1, CNE2, CNE1, and HK1 cells, Notch1 protein levels assayed by Western blot analysis from three independent experiments. WT, wild-type cells; EV, cells transfected with empty vector; Notch1-shRNA, cells transfected with Notch1-shRNA. NPC, nasopharyngeal carcinoma 
compared with those transfected with EV (Figure 2A). We further detected Hes1 expression, which is the downstream target of Notch1 signaling, and found that Hes 1 expression was dramatically reduced in Notch1 knockdown NPC cells (Figure S1A). To observe the effects of Notch1 knockdown on NPC cell growth, cell proliferation was evaluated by the MTS cell proliferation assay. CNE1 and CNE2 wildtype cells, and cells transfected with Notch1-shRNA or EV control were cultured for the indicated time points, and the results were recorded using an ELISA plate reader (Figure 2B). Lack of Notch1 expression significantly inhibited NPC cell proliferation. To elucidate the mechanism of growth inhibition in Notch1 knockdown cells, we examined cell apoptosis using the TUNEL assay. We found that cells with lower Notch1 expression had a higher apoptosis rate than EV-transfected control cells (Figure 2C). Notch1 knockdown caused 40 to $60 \%$ apoptosis whereas control cells only showed 5 to $10 \%$ apoptosis, suggesting that the growth inhibitory effects on Notch1 knockdown cells were partially because of the induction of apoptosis. Western blot results showed that the apoptotic-related proteins were
(A)

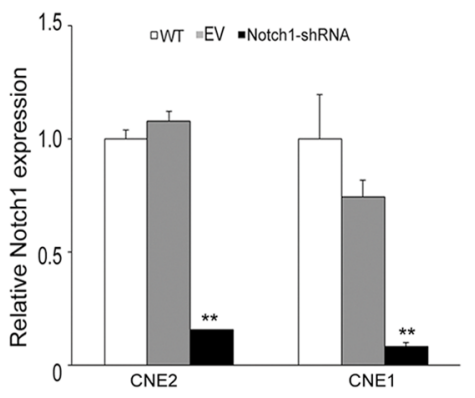

(C)

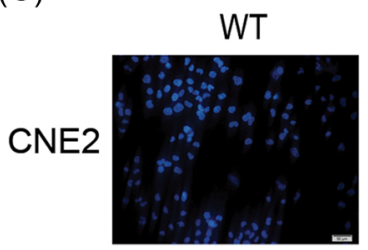

CNE1

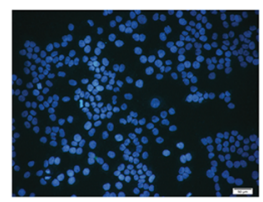

EV
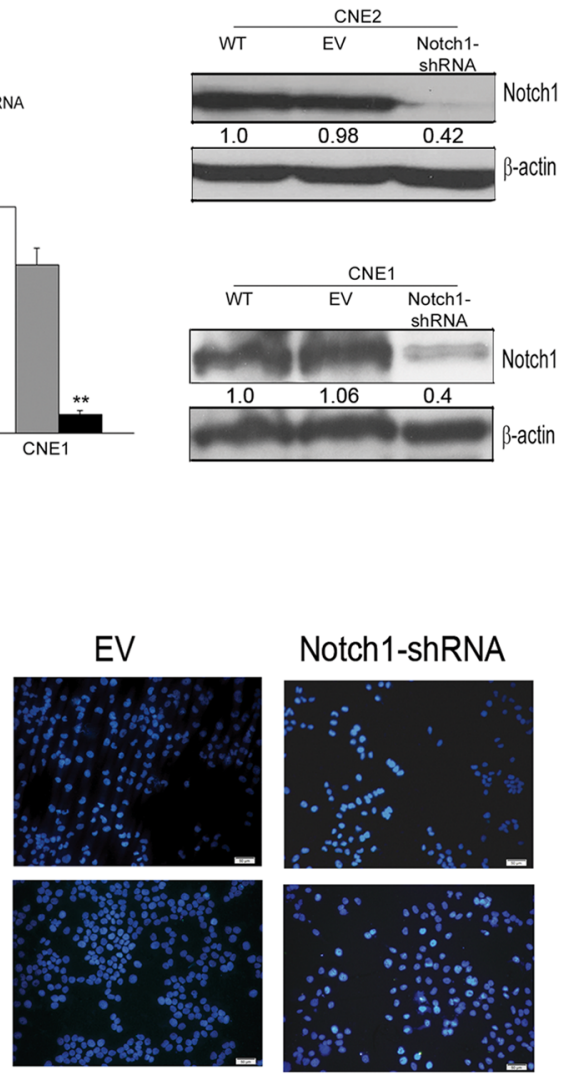

Notch1-shRNA

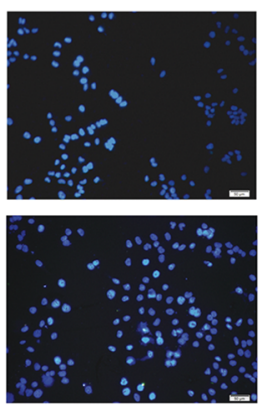

(B)
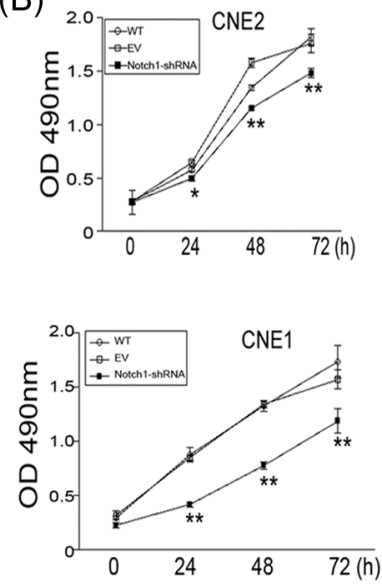

(D)

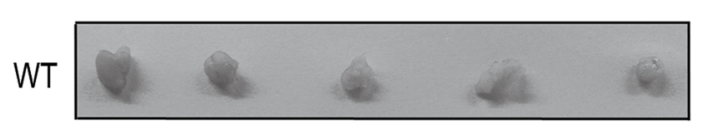

EV

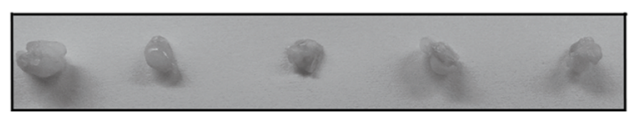

Notch1-shRNA
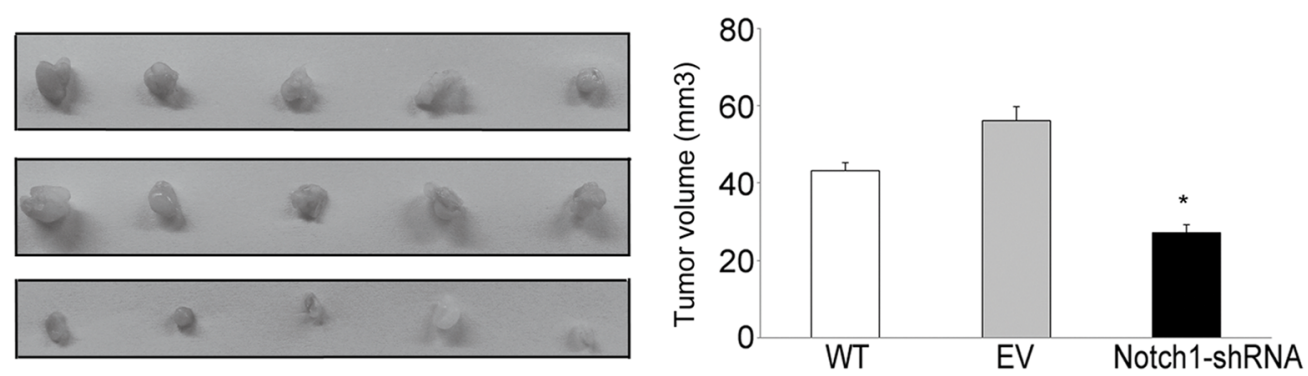

FIGURE 2 Downregulation of Notch1 expression inhibits NPC cell growth and induces apoptosis. A, The knockdown efficiency of Notch1 in CNE1 and CNE2 cells, compared with EV control cells. qPCR (left panel) and Western blot analysis (right panel) were performed. B, The proliferation of the NPC cell lines, CNE2 (upper panel) and CNE1 (lower panel), were determined by the MTS assay. C, Representative micrographs and quantitative data for NPC cell apoptosis determined by the TUNEL assay. The TUNEL assay was conducted as outlined in the Materials and Methods. Fragmented DNA was labeled in green, and all nuclei were counterstained with Hoechst 33342. Knockdown of Notch1 expression increased apoptosis about 3.5-5 folds compared with controls. D, Downregulation of Notch1 inhibited NPC tumorigenesis in the mouse model. Tumor cells were subcutaneously injected into nude mice and the tumor volume was calculated twice a week. All the data are from three separate experiments. Columns, mean of triplicates; bars, SEM. GAPDH was used as an internal control. ${ }^{*} P<.05$, and ${ }^{* *} P<.01$, respectively, indicates a significant difference compared with the EV control. EV, empty vector; NPC, nasopharyngeal carcinoma; TUNEL, terminal deoxynucleotidyl transferase dUTP nick-end labeling [Color figure can be viewed at wileyonlinelibrary.com] 
TABLE 1 Tumor formation in nude mice (CNE1 cells)

\begin{tabular}{lll} 
Group & Tumor volume, $\mathrm{mm}^{3}$ (mean \pm SEM) & $P$ \\
WT & $41.11 \pm 1.8$ & \\
EV & $56.05 \pm 3.7$ & 0.018 \\
Notch1-shRNA & $27.21 \pm 2.0$ & \\
\hline
\end{tabular}

changed. The total and cleavage Caspase9 were increased and $\mathrm{Bcl} 2$ levels were decreased in Notch1-shRNA cells (Figure S1B).

\section{3 | Downregulation of Notch1 expression inhibits CNE1 cell growth in vivo}

To explore the probability of tumor cell growth in vivo after Notch1 expression was knocked down, we subcutaneously injected CNE1 wild-type cells, the cells transfected with EV that expressed Notch1 normally, and the cells transfected with Notch1-shRNA (inhibition of Notch1 expression) into nude mice. After 4 weeks, tumors were harvested and kept in $10 \%$ formalin for further study. The results showed that knocking down Notch1 expression significantly inhibited tumorigenesis and growth compared with the controls (Figure 2D and Table 1). Together, these results indicate that the Notch1 pathway is highly expressed in NPC tissues and cell lines, and promotes tumor cell growth both in vitro and in vivo. Thus, Notch1 may be a novel valuable therapeutic target for NPC treatment.

\section{4 | Reducing Notch1 expression diminishes tumor cell migration and invasion}

To investigate the changes of biological functions in Notch1 knockdown NPC cells, we used the transwell assay to assess NPC cell migration and invasion in vitro. Cell migration was determined by counting the number of cells that penetrated from the top to bottom through a porous membrane, whereas in the cell invasion assay, cells were counted that invaded the barrier of the reconstituted matrigel layer on the membrane. Reducing Notch1 expression in CNE1 and CNE2 cells significantly diminished cell migration (CNE1 68\%, CNE2 72\%) and invasion (CNE1 35\%, CNE2 54\%) compared with wild-type cells or EV-transfected cells (Figure 3).
FIGURE 3 Knockdown of Notch1 expression diminishes NPC cell migration and invasion. A, Notch1

shRNA-transfected cells resulted in low penetration of cells through the absent or present matrigel-coated membrane, compared with the control. B,

Representative of cell migration, the cells that migrated to the lower chambers were counted. C, Cell penetration through the membrane with Matrigel was quantified by counting the numbers of cells that penetrated the membrane in five microscopic fields (at 200× magnification) per filter. Invasive index (\%) was defined as the proportion of cells that penetrated the Matrigel-coated membrane divided by the number of cells that migrated through the uncoated membrane (baseline migration). Columns, mean of triplicate assays; bars, SEM. ${ }^{*} P<.05$, and ${ }^{* *} P<.01$, respectively, indicates a significant difference compared with the EV control. All the data are from three separate experiments. EV, empty vector; NPC, nasopharyngeal carcinoma [Color figure can be viewed at wileyonlinelibrary.com]
(A)
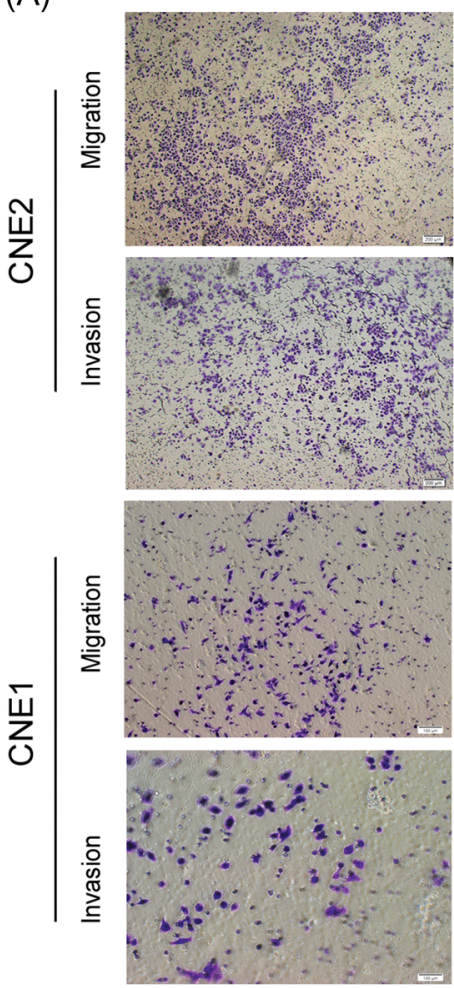

(B)

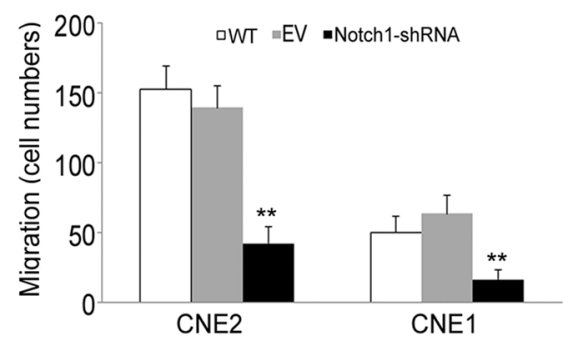

EV
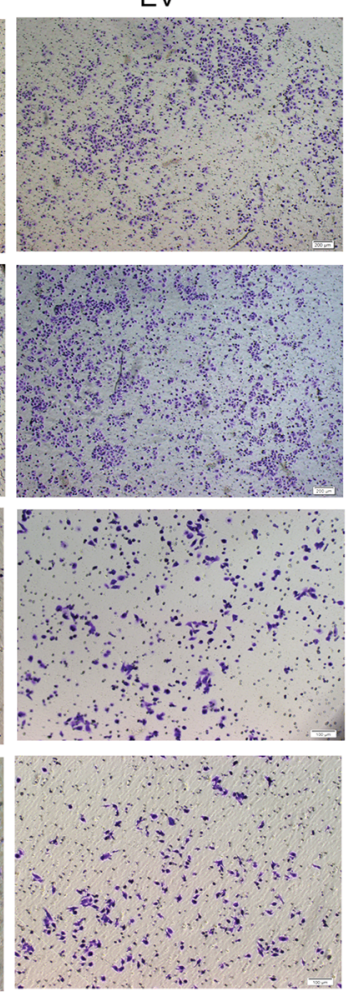

(C)

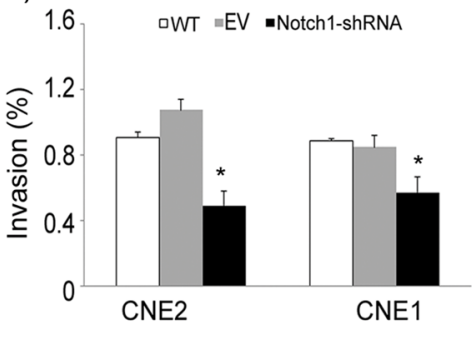

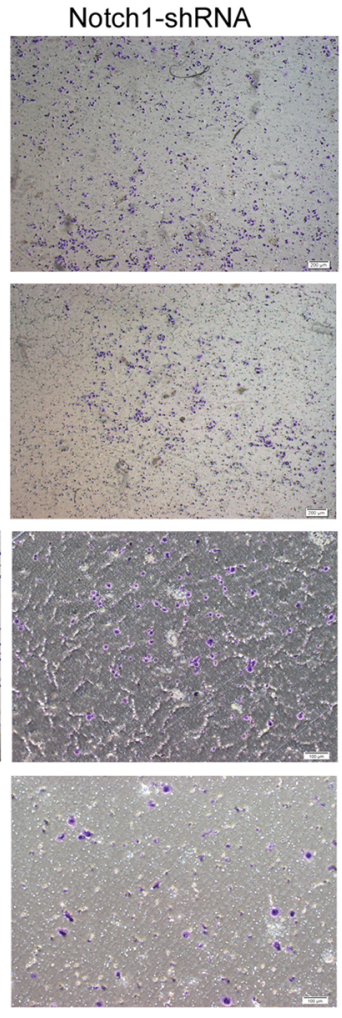




\subsection{Inhibition of Notch1 expression reverses the EMT in NPC cells}

We observed that the morphology of NPC cells changed from spindle-shaped into epithelioid-like cells when Notch1 expression was decreased (Figure 4A). To determine the molecular mechanisms underlying the correlation of Notch1 expression with the EMT in cancer cells, we monitored EMT marker changes in NPC wild-type cells and cells transfected with Notch1-shRNA or EV by Western blot analysis. We found that inhibition of Notch1 in both CNE2 and CNE1 cells significantly enhanced $\mathrm{E}$-cadherin and $\beta$-catenin expression and decreased vimentin expression (Figure 4B). These results indicate that Notch1 knockdown might disrupt the migration and invasion of NPC cells by reversing EMT alterations in cancer cells.

\subsection{Knockdown of Notch1 decreases CCL2 and CXCL16 secretion}

We previously demonstrated that downregulation of CCL2 or CXCL16 significantly diminishes prostate cancer (PCa) tumor growth both in vitro and in vivo. ${ }^{35-37}$ Knocking down CCL2 production in $\mathrm{PCa}$ cells notably reduces PCa CM-induced osteoclast formation in vitro. $^{36}$ Bone metastasis is a very common event in patients with advanced NPC and PCa and is also the main cause of death in NPC.
To extend these observations, we determined if Notch1 knockdown in NPC cell clones affected the expression of CCL2 and CXCL16. In Notch1 knockdown cells, the expression of CCL2 and CXCL16 was markedly inhibited (Figure 5A,B), indicating that they might be downstream targets of Notch1 in patients with advanced NPC. When CCL2 expression was knocked down ${ }^{38}$ in CNE1 cells, the level of Notch1 did not show apparent change. Further, we overexpressed active Notch1 intracellular domain (NICD-Ov) in CNE1 cells, collected the supernatants from either NICD-Ov cells or control cells. The CCL2 expression level was significantly increased in NICDOv cells (Figure S2).

\subsection{Knockdown of Notch1 reduces the production of UPA and UPAR}

It had been reported that uPA might act as a downstream target of Notch signaling in $\mathrm{PCa} .{ }^{39}$ Hence, to further explore the potential mechanisms of Notch1 knockdown on invasion inhibition of tumor cells, we examined the protein levels of UPA and UPAR by ELISA. We found that the soluble uPAR was markedly reduced in Notch1 knockdown cells compared with the EV control (Figure 5C). The protein level of UPA in CNE1 cells was also significantly decreased after Notch1 knockdown (Figure 5D). However, uPA expression in
(A)
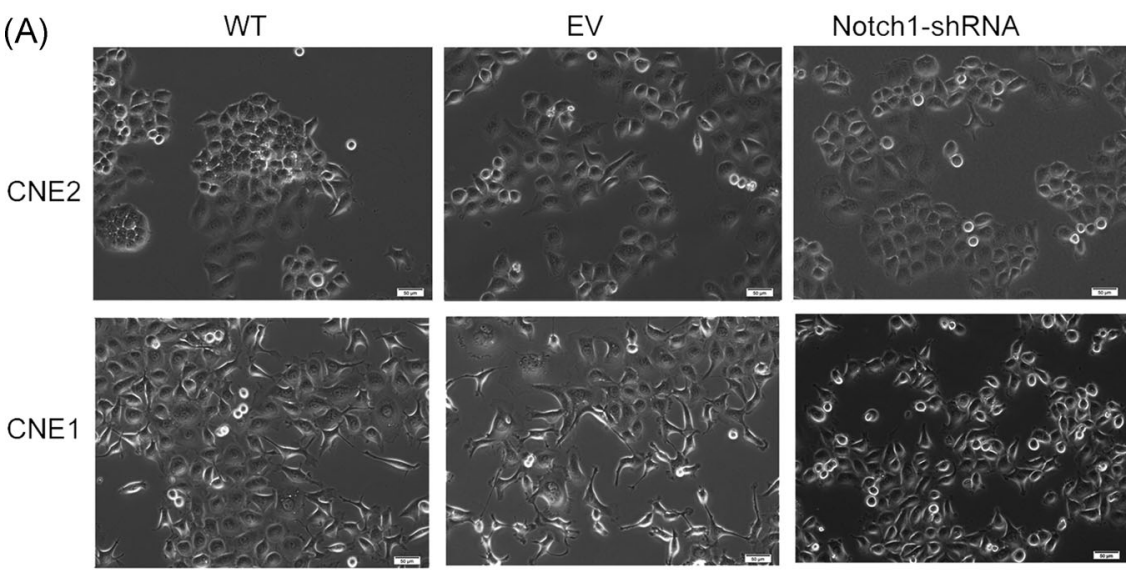

(B)

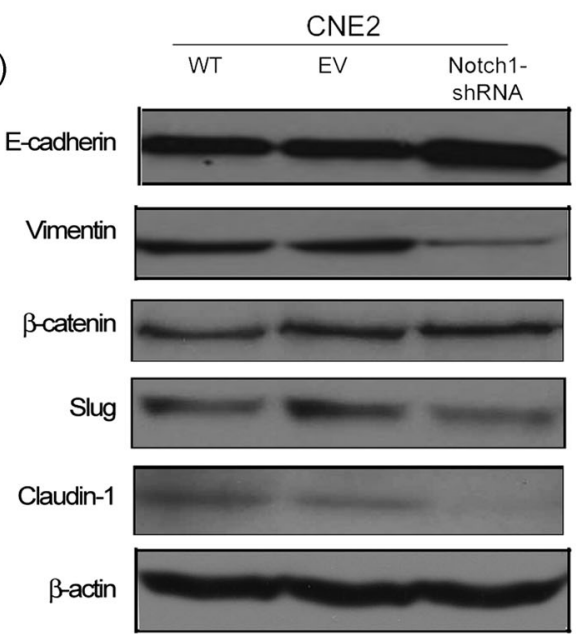

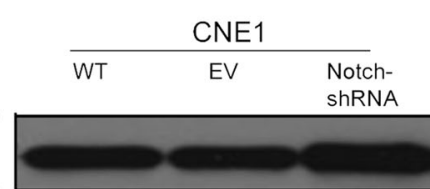
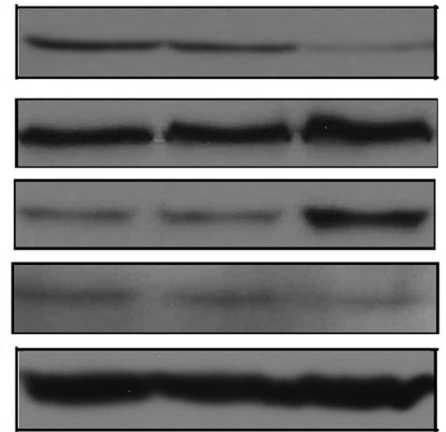

FIGURE 4 Knockdown of Notch1 expression in NPC cells inhibits the EMT. A, Knockdown of Notch1 leads to cell morphology changes. Top, morphological changes in CNE2 cells; bottom, the morphology of CNE1 cells changed from spindle-shaped into epithelioid-like cells. B, Notch1 shRNA-transfected cells resulted in the significant upregulation of Ecadherin protein expression and a reduction of Vimentin and Slug expression levels from three

independent experiments. EMT, epithelialmesenchymal transition; EV, empty vector; NPC, nasopharyngeal carcinoma 
(A)

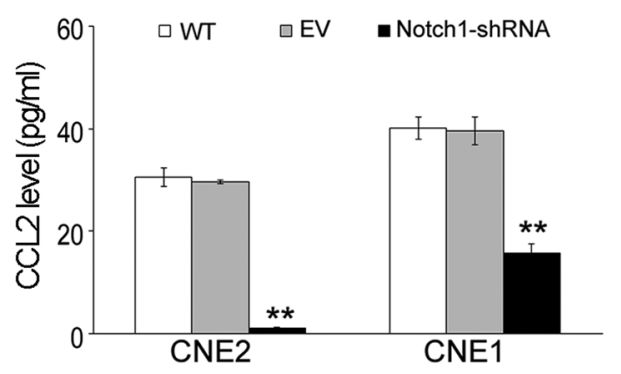

(C)

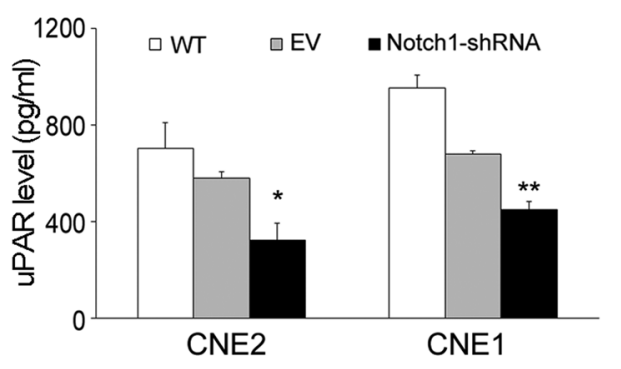

(B)

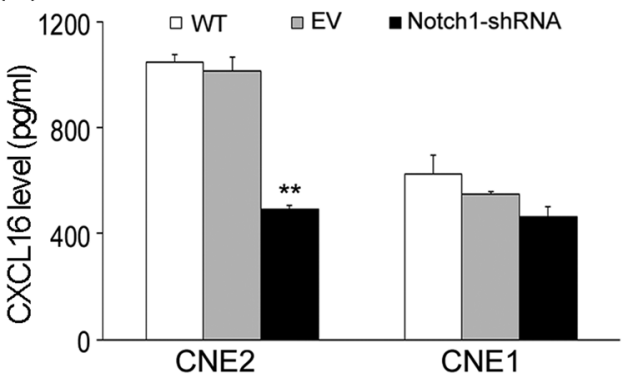

(D)

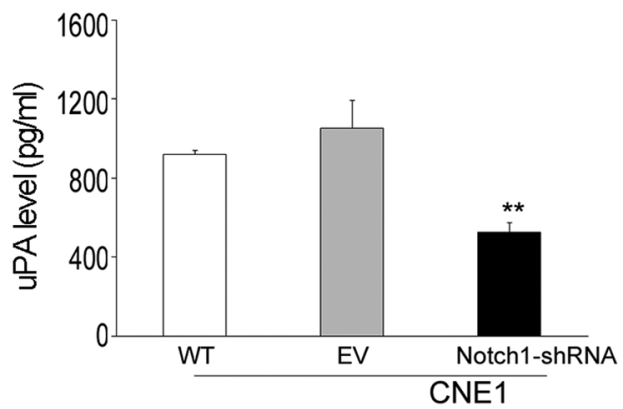

FIGURE 5 Inhibition of Notch1 expression decreases the production of CCL2, CXCL16, uPA, and uPAR. A, The expression of CCL2 protein was significantly inhibited in Notch1 shRNA-transfected cells. B, The expression of CXCL16 in CNE2 cells was decreased by more than half in Notch1 shRNA-transfected cells compared with the control cells. In CNE1 cells, knockdown of Notch1 expression also led to a decrease of CXCL16, but the result was not significantly different. C, The expression of the uPAR protein was markedly inhibited in Notch1 shRNA-transfected cells. D, Knockdown of Notch1 decreased uPA expression by about two-fold. Columns, mean of triplicate assays; bars, SEM. " and " represent $P<.05$, and $P<.01$, respectively, indicates a significant difference compared with the EV control. All the data are from at least three independent experiments. EV, empty vector; uPAR, urokinase plasminogen activator receptor

CNE2 cells was too low to detect and was also undetectable in Notch1 knockdown cells. Together, these results indicate that Notch1 regulation of UPA and UPAR secretion may be a mechanism, at least in part, for the migration and invasion of NPC cells.

\section{8 | uPA acts as a key mediator in Notch1-induced NPC cell proliferation and invasion}

To confirm the effects of uPA in Notch1-induced NPC cell proliferation and invasion, we did a literature search in the GEO database, ${ }^{34}$ and found that UPA gene expression levels were much higher in NPC tissues than in normal nasopharyngeal tissues (Figure 6A). ${ }^{34}$ Using qPCR and Western blot, we observed that uPA expression level was greater in CNE1 and HK1 cells than in HNEC or CNE2 cells (Figure S3). Furthermore, we downregulated uPA expression in CNE1 cells by UPA-shRNA. The uPA gene expression was evaluated using qPCR in CNE1 wild-type cells, and cells transfected with EV or uPA-shRNA (Figure 6B). In functional studies, downregulation of uPA expression markedly retarded tumor cell growth, decreased cyclin $D$ and cyclin $E$ protein levels, and reversed the EMT. Knockdown of uPA expression significantly reduced tumor cell invasion (Figure 6C-E). Taken together, these results indicate that knockdown of Notch1 expression significantly reduces uPA production, and that downregulation of UPA has similar effects on tumor cell growth, progression, and metastasis.

\section{4 | DISCUSSION}

Dysregulation of Notch signaling has been detected in various types of human cancers, including breast, ${ }^{13,15}$ lung, $^{22,23}$ and brain cancers. ${ }^{40}$ It has also been reported that Notch signaling positively correlates with the proliferation of NPC. Suppression of all Notch receptors (Notch1-4) using GSI ( $\gamma$-secretase inhibitor) inhibit human NPC cell proliferation ${ }^{37}$ and enhances the radiosensitivity of NPC cells. ${ }^{41}$ In this study, we analyzed the impact of Notch1 on the onset and progression of NPC. We first determined Notch1 expression levels in different NPC cell lines (HONE1, SUNE1, CNE1, CNE2, and HK1) and normal HNEC, and observed that all the tested NPC cell lines expressed a higher level of Notch1 mRNA and protein compared with the normal cells. We chose CNE1 and CNE2 cell lines for further studies, as Notch1 protein levels were more highly expressed in those cell lines. We found that the targeted disruption of Notch1 in CNE1 and CNE2 cells by shRNA resulted in significant inhibition of cell proliferation and induction of apoptosis. Downregulation of Notch1 decreased cell invasion across the artificial matrix, which mimics the in vivo extracellular matrix. Thus, our results provide in vitro evidence to support the role of Notch1 as an oncogene rather than a tumor suppressor gene in NPC cells. Furthermore, the downregulation of Notch1 inhibits tumor formation and growth in vivo. 
(A)

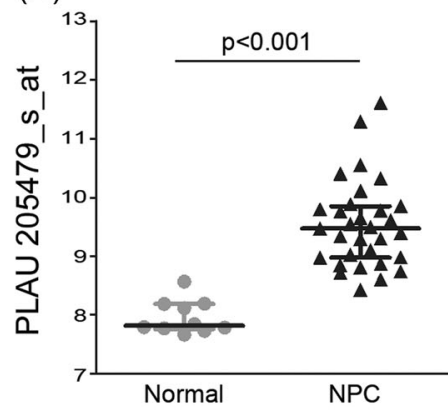

(D)

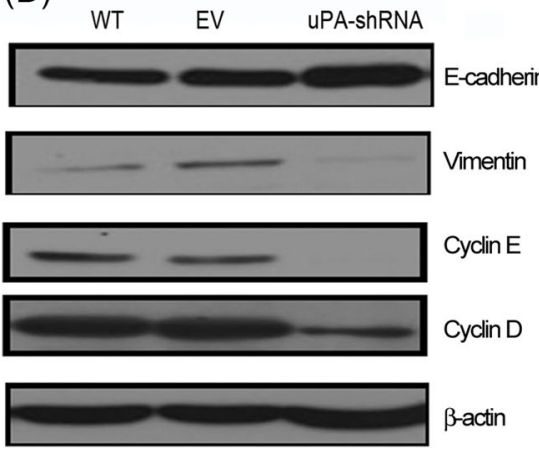

(B)

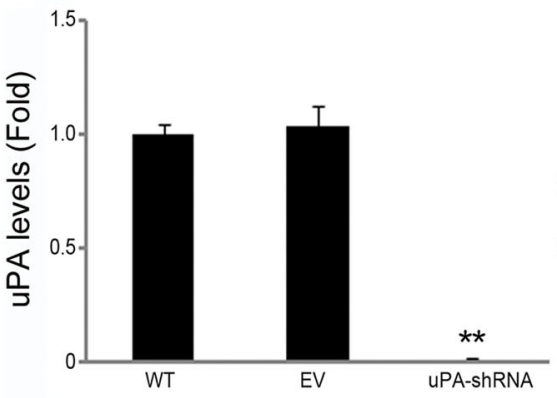

(C)

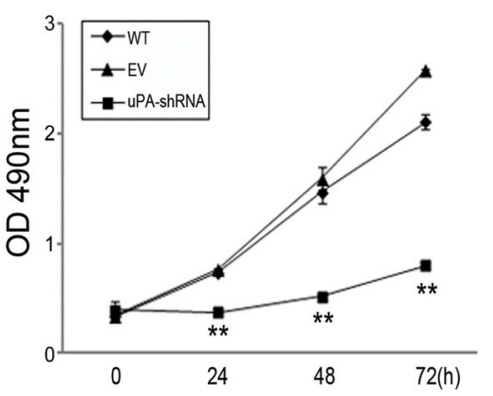

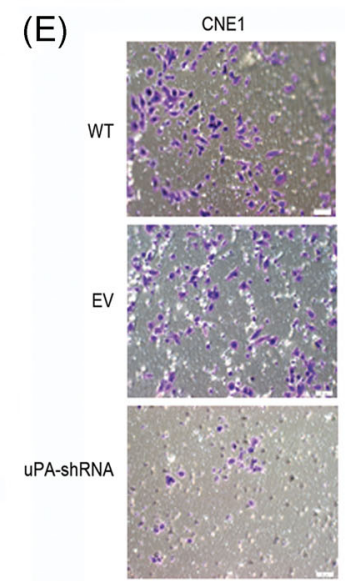

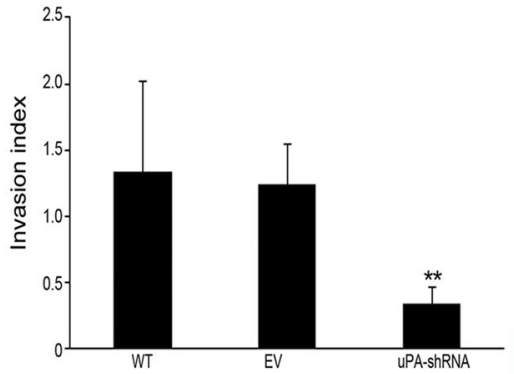

FIGURE 6 uPA acts as a key mediator in Notch1-induced NPC cell proliferation and invasion. A, The scatterplot shows that uPA gene was highly expressed in NPC tissues by Human Genome U133 Plus 2.0 Array. ${ }^{34}$ B, The knockdown efficiency of uPA in CNE1 cells, compared with EV control cells, was determined by qPCR. C, CNE1 cell proliferation was examined by the MTS assay. D, uPA-shRNA-transfected cells resulted in the significant upregulation of E-cadherin protein expression and a reduction in vimentin expression. Cyclin $D$ and cyclin $E$ expression were markedly decreased in uPA knockdown cells. E, uPA-shRNA-transfected cells (left panel) resulted in the low penetration of cells through the Matrigel-coated membrane, compared with control cells. Invasive Index (\%) was calculated (right panel) according to the manufacturer's instructions. The data are from three independent experiments. Columns, mean of triplicate assays; bars, SEM. EV, empty vector; NPC, nasopharyngeal carcinoma; shRNA, short hairpin RNA; uPA, urokinase plasminogen activator. ${ }^{*} P<.05$, and ${ }^{* *} P<.01$, respectively, indicates a significant difference compared with the EV control [Color figure can be viewed at wileyonlinelibrary.com]

The EMT is involved in the metastasis and progression of tumors. ${ }^{42,43}$ The most representative marker in the process of epithelial morphogenesis is E-cadherin expression, as the repression and/or delocalization of E-cadherin is usually associated with adherent junctions disassembly ${ }^{44}$ and enhanced cell invasiveness. ${ }^{45}$ The activation of Notch signaling contributes to the acquisition of the EMT in both breast ${ }^{46}$ and pancreatic cancers. ${ }^{47}$ In this study, we found that Notch1 knockdown markedly diminishes NPC cell migration and invasion. We also observed the upregulation of E-cadherin protein expression along with the reduction of Vimentin levels in Notch1 knockdown cells.

In addition to EMT effector molecules controlling the cell invasion and metastasis, the UPA/UPAR axis has been implicated in the destruction of the basement membrane and extracellular matrix, which is associated with tumor cell invasion. ${ }^{33,48,49}$ Several studies have reported that the downregulation of Notch1 or Jagged1 decreases the expression and activity of uPA, which contributes to the inhibition of cancer cell migration, invasion, and apoptosis in glioblastoma, ${ }^{50}$ breast cancer, ${ }^{51}$ and PCa cells. ${ }^{39}$ Here, we provided evidence that the Notch1 pathway might associate closely with the uPA/UPAR axis. Using qPCR and Western blot, we observed that uPA expression level was greater in CNE1 and HK1 cells than in HNEC or CNE2 cells. These results prompt that uPA levels may play an important role in nasopharyngeal carcinogenesis. In agreement with others, we showed that uPA transcription was downregulated in Notch1-specific knockdown cells. Overall, these results suggest that knockdown Notch1 attenuates cell migration and invasion, at least in part, by inhibiting the expression and activity of the uPA/uPAR axis. To the best of our knowledge, this is the first report to demonstrate a direct link between activation of the uPA/uPAR axis and Notch signaling in NPC development.

We also observed that the Notch1 pathway affected the secretion of chemokines CCL2 and CXCL16 in NPC cells. Notch signaling directly regulates the expression of two powerful proinflammatory cytokines, interleukin 1 beta, and CCL2, in basal-like breast cancer, leading to the recruitment of tumor-associated macrophages to support tumor growth and metastasis. ${ }^{52}$ Previous works have reported that CCL2 is associated with $\mathrm{PCa}$ bone metastasis. ${ }^{36,38}$ CCL2 knockdown using shRNA approach diminished PCa cell invasion and PCa CM-induced osteoclast formation. 
Similarly, recent evidence from Xiang's group indicated that the serum concentration of CCL2 in patients with NPC with large skull base invasion were higher than those without or with small skull invasion. ${ }^{53}$ Collectively, these works highlight the promoting effect of $\mathrm{CCL} 2$ in tumor bone metastasis. In this study, the reduction of $\mathrm{CCL} 2$ and CXCL16 expression in Notch1 knockdown cells was observed, suggesting that Notch1 can also regulate the production of CCL2 and CXCL16, which might be involved with advanced NPC progression. We further knocked down CCL2 expression in CNE1 cells, the level of Notch1 did not show apparent change. Moreover, overexpression of NICD in CNE1 cells significantly increased CCL2 level compared with the control cells. Additional studies on the mechanisms of Notch1 and CCL2 in NPC bone metastasis are warranted.

\section{5 | CONCLUSIONS}

This is the first report to explore the role of Notch1 in the onset and progression of NPC. We showed that Notch1 knockdown reduced NPC cell migration and invasion in vitro by reversing the EMT, and decreasing the expression of UPA/UPAR and the chemokines CCL2 and CXCL16. These results indicate that the Notch1 gene may be a novel therapeutic target for NPC treatment.

\section{ACKNOWLEDGMENTS}

The authors thank Jiejun Fu and Qiong Song for technical assistance and helpful discussions. We also thank Xia Liu and Xin Huang for editing.

This work was supported by Science and Technology Foundation of Shenzhen Grants JCYJ2017030711041760 and JCYJ20170307105128101; National Natural Science Foundation of China (NSFC) 81773146, NSFC Key Project 81130046; Guangdong Provincial Key Laboratory of Cell Microenvironment and Disease Research 2017B030301018; Guangxi Natural Science Foundation $2015 G X N S F B A 139117$.

\section{CONFLICT OF INTEREST}

The authors declare that there is no conflict of interest.

\section{ORCID}

Yi Lu (D) http://orcid.org/0000-0002-1060-6925

\section{REFERENCES}

1. Wildeman MA, Fles R, Herdini $C$, et al. Primary treatment results of Nasopharyngeal Carcinoma (NPC) in Yogyakarta, Indonesia. PLoS One. 2013;8:e63706.

2. Tang LL, Chen WQ, Xue WQ, et al. Global trends in incidence and mortality of nasopharyngeal carcinoma. Cancer Lett. 2016; 374:22-30.
3. Peng H, Chen L, Chen YP, et al. The current status of clinical trials focusing on nasopharyngeal carcinoma: a comprehensive analysis of ClinicalTrials.gov database. PLoS One. 2018;13:e0196730.

4. Lee AW, Lin JC, Ng WT. Current management of nasopharyngeal cancer. Semin Radiat Oncol. 2012;22:233-244.

5. Hu W, Ding W, Yang H, et al. Weekly paclitaxel with concurrent radiotherapy followed by adjuvant chemotherapy in locally advanced nasopharyngeal carcinoma. Radiother Oncol. 2009;93:488-491.

6. Chua MLK, Wee JTS, Hui EP, et al. Nasopharyngeal carcinoma. Lancet. 2016;387:1012-1024.

7. Morgan TH. Goodale's experiments on gonadectomy of fowls. Science. 1917;45:483-484.

8. Artavanis-Tsakonas S, Rand MD, Lake RJ. Notch signaling: cell fate control and signal integration in development. Science. 1999;284: 770-776.

9. Gray GE, Mann RS, Mitsiadis E, et al. Human ligands of the Notch receptor. Am J Pathol. 1999;154:785-794.

10. Pannuti A, Foreman K, Rizzo P, et al. Targeting notch to target cancer stem cells. Clin Cancer Res. 2010;16:3141-3152.

11. Ranganathan $P$, Weaver $K L$, Capobianco AJ. Notch signalling in solid tumours: a little bit of everything but not all the time. Nat Rev Cancer. 2011;11:338-351.

12. Ellisen LW, Bird J, West DC, et al. TAN-1, the human homolog of the Drosophila notch gene, is broken by chromosomal translocations in T lymphoblastic neoplasms. Cell. 1991;66:649-661.

13. Sethi N, Dai X, Winter CG, et al. Tumor-derived JAGGED1 promotes osteolytic bone metastasis of breast cancer by engaging notch signaling in bone cells. Cancer Cell. 2011;19:192-205.

14. Farnie G, Clarke RB. Mammary stem cells and breast cancer-role of Notch signalling. Stem Cell Rev. 2007;3:169-175.

15. Stylianou S, Clarke RB, Brennan K. Aberrant activation of notch signaling in human breast cancer. Cancer Res. 2006;66:1517-1525.

16. Steg AD, Katre AA, Goodman B, et al. Targeting the notch ligand JAGGED1 in both tumor cells and stroma in ovarian cancer. Clin Cancer Res. 2011;17:5674-5685.

17. McAuliffe SM, Morgan SL, Wyant GA, et al. Targeting notch, a key pathway for ovarian cancer stem cells, sensitizes tumors to platinum therapy. Proc Natl Acad Sci USA. 2012;109:E2939-E2948.

18. Xiao W, Gao Z, Duan Y, et al. Notch signaling plays a crucial role in cancer stem-like cells maintaining stemness and mediating chemotaxis in renal cell carcinoma. J Exp Clin Cancer Res. 2017;36:41.

19. Liu ZJ, Xiao M, Balint $\mathrm{K}$, et al. Notch1 signaling promotes primary melanoma progression by activating mitogen-activated protein kinase/phosphatidylinositol 3-kinase-Akt pathways and up-regulating N-cadherin expression. Cancer Res. 2006;66:4182-4190.

20. Reedijk M, Odorcic S, Chang L, et al. High-level coexpression of JAG1 and NOTCH1 is observed in human breast cancer and is associated with poor overall survival. Cancer Res. 2005;65:8530-8537.

21. Nicolas $M$, Wolfer $A$, Raj $K$, et al. Notch1 functions as a tumor suppressor in mouse skin. Nature Genet. 2003;33:416-421.

22. Hassan KA, Wang L, Korkaya $H$, et al. Notch pathway activity identifies cells with cancer stem cell-like properties and correlates with worse survival in lung adenocarcinoma. Clin Cancer Res. 2013; 19:1972-1980.

23. Donnem T, Andersen S, Al-Shibli K, et al. Prognostic impact of Notch ligands and receptors in nonsmall cell lung cancer: coexpression of Notch-1 and vascular endothelial growth factor-A predicts poor survival. Cancer. 2010;116:5676-5685.

24. Sriuranpong V, Borges MW, Ravi RK, et al. Notch signaling induces cell cycle arrest in small cell lung cancer cells. Cancer Res. 2001;61: 3200-3205.

25. Li J, Li Q, Lin L, et al. Targeting the Notch1 oncogene by miR-139-5p inhibits glioma metastasis and epithelial-mesenchymal transition (EMT). BMC Neurol. 2018;18:133. 
26. Teng ZP, Ooka T, Huang DP, et al. Detection of Epstein-Barr virus DNA in well and poorly differentiated nasopharyngeal carcinoma cell lines. Virus Genes. 1996;13:53-60.

27. Yan Min, Zhang Yan, et al. IKKa restoration via EZH2 suppression induces nasopharyngeal carcinoma differentiation. Nat Commun. 2014;5:3661. https://doi.org/10.1038/ncomms4661.

28. Lu Y, Cai Z, Galson DL, et al. Monocyte chemotactic protein-1 (MCP1) acts as a paracrine and autocrine factor for prostate cancer growth and invasion. Prostate. 2006;66:1311-1318.

29. Lu Y, Wang J, Xu Y, et al. CXCL16 functions as a novel chemotactic factor for prostate cancer cells in vitro. Mol Cancer Res. 2008;6:546-554.

30. Lu Y, Cai Z, Xiao G, et al. CCR2 expression correlates with prostate cancer progression. J Cell Biochem. 2007;101:676-685.

31. Lu Y, Xiao G, Galson DL, et al. PTHrP-induced MCP-1 production by human bone marrow endothelial cells and osteoblasts promotes osteoclast differentiation and prostate cancer cell proliferation and invasion in vitro. Int J Cancer. 2007;121:724-733.

32. Lu Y, Nie D, Witt WT, et al. Expression of the fat-1 gene diminishes prostate cancer growth in vivo through enhancing apoptosis and inhibiting GSK-3 beta phosphorylation. Mol Cancer Ther. 2008;7: 3203-3211.

33. Zhang J, Sud S, Mizutani K, et al. Activation of urokinase plasminogen activator and its receptor axis is essential for macrophage infiltration in a prostate cancer mouse model. Neoplasia. 2011;13:23-30.

34. Sengupta S, den Boon JA, Chen IH, et al. Genome-wide expression profiling reveals EBV-associated inhibition of $\mathrm{MHC}$ class I expression in nasopharyngeal carcinoma. Cancer Res. 2006;66:7999-8006.

35. Lu Y, Cai Z, Xiao G, et al. Monocyte chemotactic protein-1 mediates prostate cancer-induced bone resorption. Cancer Res. 2007;67: 3646-3653.

36. Yu S, Zhang R, Liu F, et al. Down-regulation of Notch signaling by a gamma-secretase inhibitor enhances the radiosensitivity of nasopharyngeal carcinoma cells. Oncol Rep. 2011;26:1323-1328.

37. Chen SM, Liu JP, Zhou JX, et al. Suppression of the notch signaling pathway by gamma-secretase inhibitor GSI inhibits human nasopharyngeal carcinoma cell proliferation. Cancer Lett. 2011;306:76-84.

38. Lu Y, Chen Q, Corey E, et al. Activation of MCP-1/CCR2 axis promotes prostate cancer growth in bone. Clin Exp Metastasis. 2009; 26:161-169.

39. Bin Hafeez B, Adhami VM, Asim M, et al. Targeted knockdown of Notch1 inhibits invasion of human prostate cancer cells concomitant with inhibition of matrix metalloproteinase- 9 and urokinase plasminogen activator. Clin Cancer Res. 2009;15:452-459.

40. Fan X, Matsui W, Khaki L, et al. Notch pathway inhibition depletes stem-like cells and blocks engraftment in embryonal brain tumors. Cancer Res. 2006;66:7445-7452.

41. Thiery JP, Acloque H, Huang RY, et al. Epithelial-mesenchymal transitions in development and disease. Cell. 2009;139:871-890.

42. Marambaud P, Shioi J, Serban G, et al. A presenilin-1/gamma-secretase cleavage releases the E-cadherin intracellular domain and regulates disassembly of adherens junctions. EMBO J. 2002;21:1948-1956.
43. Kalluri R, Weinberg RA. The basics of epithelial-mesenchymal transition. J Clin Invest. 2009;119:1420-1428.

44. Peinado H, Portillo F, Cano A. Transcriptional regulation of cadherins during development and carcinogenesis. Int J Dev Biol. 2004;48: 365-375.

45. Bolos V, Mira E, Martinez-Poveda B, et al. Notch activation stimulates migration of breast cancer cells and promotes tumor growth. Breast Cancer Res. 2013;15:R54.

46. LeBeau AM, Duriseti S, Murphy ST, et al. Targeting uPAR with antagonistic recombinant human antibodies in aggressive breast cancer. Cancer Res. 2013;73:2070-2081.

47. Ni X, Long J, Cen P, et al. Pancreatic cancer tumour initiating cells: the molecular regulation and therapeutic values. J Cell Mol Med. 2012;16:988-994.

48. Raghu H, Gondi CS, Dinh DH, et al. Specific knockdown of uPA/uPAR attenuates invasion in glioblastoma cells and xenografts by inhibition of cleavage and trafficking of Notch -1 receptor. Mol Cancer. 2011; 10:130.

49. Mahmood N, Mihalcioiu C, Rabbani SA. Multifaceted role of the Urokinase-Type Plasminogen Activator (UPA) and its receptor (UPAR): diagnostic, prognostic, and therapeutic applications. Front Oncol. 2018;8:24.

50. Fassl A, Tagscherer KE, Richter J, et al. Notch1 signaling promotes survival of glioblastoma cells via EGFR-mediated induction of antiapoptotic Mcl-1. Oncogene. 2012;31:4698-4708.

51. Shimizu M, Cohen B, Goldvasser P, et al. Plasminogen activator UPA is a direct transcriptional target of the JAG1-Notch receptor signaling pathway in breast cancer. Cancer Res. 2011;71:277-286.

52. Shen Q, Cohen B, Zheng W, et al. Notch shapes the innate immunophenotype in breast cancer. Cancer Discov. 2017;7:1320-1335.

53. Lu X, Qian CN, Mu YG, et al. Serum CCL2 and serum TNF- $\alpha$-two new biomarkers predict bone invasion, post-treatment distant metastasis and poor overall survival in nasopharyngeal carcinoma. Eur J Cancer. 2011;47:339-346.

\section{SUPPORTING INFORMATION}

Additional supporting information may be found online in the Supporting Information section.

How to cite this article: Guo H, Wang F, Diao Y, et al. Knockdown of Notch1 inhibits nasopharyngeal carcinoma cell growth and metastasis via downregulation of CCL2, CXCL16, and uPA. Molecular Carcinogenesis. 2019;58:1886-1896. https://doi.org/10.1002/mc.23082 